\title{
Laser-based precision magnetometry in fundamental and applied research
}

\author{
A. Weis*, R. Wynands ${ }^{1}$ \\ Département de Physique, Université de Fribourg, Chemin du Musée 3, 1700 Fribourg, Switzerland
}

Received 4 December 2003; received in revised form 16 March 2004; accepted 24 March 2004

Available online 27 July 2004

\begin{abstract}
In this paper we review research done by the recently founded Fribourg group for Atomic Physics (FRAP) using laser-assisted spin coherence spectroscopy of atomic samples in applied and fundamental physics. Spin coherence in optically pumped ensembles of paramagnetic atoms may be extremely long-lived when the samples are well shielded from external perturbations, like static or oscillating magnetic fields and field gradients, static electric fields, or crystalline field gradients. Conversely, such interactions may be studied with high sensitivity and precision when the decoherence that they produce is measured by optical means. We report on applications of this technique covering optical magnetometry for biomagnetic diagnostics, investigations of atomic impurities in quantum crystals, measurements of forbidden tensor polarizabilities and electric dipole moments, and the space-time resolved study of the diffusion of gas-phase atoms.
\end{abstract}

Keywords: Optical magnetometry; Spin physics; Cardiomagnetometry; Magnetocardiogram; Solid helium; Electric dipole moment; Decoherence

\section{Introduction}

The research of the Fribourg Group for Atomic Physics (FRAP), installed in 2000, is centered on high-resolution atomic spectroscopy using double resonance techniques, involving laser light in combination with radio-frequency and micro-wave

*Corresponding author.

E-mail address: antoine.weis@unifr.ch (A. Weis).

${ }^{1}$ Present address: Physikalisch-Technische Bundesanstalt, 38116 Braunschweig, Germany. 
radiation. Our research addresses fundamental problems, such as investigations of helium matrix-isolated atoms, precision measurements of strongly suppressed processes or violations of discrete symmetries, as well as areas of applied research. A common tool in all investigations is the use of long-lived atomic spin coherences as a sensitive probe to detect the interaction of low-density atomic samples with their environment. Alterations of the coherence properties by controlled external perturbations can be used to measure specific atomic properties. Conversely, atomic coherences with well-known properties can be used as probes for highly sensitive detection and quantitative measurements of external perturbations. In this paper we present an overview of FRAP activities in the development and application of optical magnetometers, a straightforward application of high-resolution spectroscopy with spin-coherent atoms. Here we mostly deal with the engineering aspects of optical magnetometry, but we also take the opportunity to present a number of other applications, in which we apply optical magnetometry in fundamental research.

\section{Atomic spin coherences and their manipulation}

An ensemble of atoms with total atomic angular momentum $\vec{F}=\vec{J}+\vec{I}$, where $\vec{J}$ and $\vec{I}$ are the total electronic and nuclear angular momenta, respectively, is said to be unpolarized if all magnetic sublevels $\left|F, m_{F}\right\rangle$ with $-F \leqslant m_{F} \leqslant F$ are populated according to the Boltzmann distribution in thermodynamic equilibrium. In the case of degenerate levels this means that all levels are equally populated and that there are no coherences between these sublevels. The density matrix describing such an ensemble is diagonal and all matrix elements are equal. Ensembles whose density matrix differs from the one mentioned are called polarized. In particular, an ensemble with non-zero off-diagonal matrix elements is said to carry spin coherence or to be transversely polarized.

The angular momentum $\vec{F}$ can couple to static and oscillating magnetic fields $\vec{B}$ via the associated magnetic moment

$$
\vec{\mu}=\frac{g_{F} \mu_{B}}{\hbar} \vec{F}
$$

contained in the Zeeman Hamiltonian

$$
H=-\vec{\mu} \cdot \vec{B},
$$

where the strength of the coupling is conveniently described by the Larmor frequency

$$
\omega_{\mathrm{L}}=\frac{\vec{\mu} \cdot \vec{B}}{\hbar} .
$$

The simplest and best-known dynamic process mediated by such interactions is the Larmor precession of spins in a homogeneous magnetic field. In an inhomogeneous magnetic field, atoms located at different positions precess at different Larmor frequencies and the Larmor frequency spectrum shows a corresponding inhomogeneous broadening. Spins can also be manipulated by the combination of a static field 
$B_{0}$ and a time-varying field $B_{1}(t)$ oscillating at the frequency $\omega_{\text {rf }}$, a technique known as magnetic resonance (MR). When $\omega_{\mathrm{rf}}$ is scanned, a resonant depolarization of the sample occurs when $\omega_{\text {rf }}$ coincides with the Larmor frequency $\omega_{0}$ of the static field $B_{0}$. The ultimate width of this resonance and hence the spectral resolution is determined by the lifetime $T_{2}$ of the spin coherence. In conventional MR experiments the resonance is detected by measuring directly the magnetic field radiated by the precessing spins, thus limiting the technique to high-density samples. If, however, the MR technique is combined with optical detection, it can be applied to lowdensity samples, such as room-temperature alkali vapors.

Electric fields cannot couple directly to magnetic moments. However, electric fields and field gradients applied to an atom will mix states of different angular momentum quantum numbers $L$, thereby affecting the orbital magnetic moments $\mu_{L}$. This mixing, together with the spin-orbit interaction (proportional to $\vec{L} \cdot \vec{S}$ ) and with the hyperfine interaction (proportional to $\vec{J} \cdot \vec{I}$ ) then make that electric fields couple in an indirect way to the atomic angular momenta $\vec{J}$ and $\vec{F}$. We use these $E$-field and $E$-field gradient-induced alterations of spin coherences for the investigation of static and dynamic trapping site effects on paramagnetic atoms doped into helium crystals or for the measurement of forbidden tensor polarizabilities. An electric field can also couple directly to the atomic spin if the atom has a permanent electric dipole moment (edm). Such moments are forbidden by fundamental conservation laws, and MR experiments in electric fields can therefore be used to search for such moments, i.e., to search for a violation of these laws and the underlying discrete symmetries.

\section{Optical preparation and detection of atomic spin coherence}

Fig. 1 shows the general principle of spin coherence experiments with optical preparation and detection. Resonant or near resonant laser light is used both to prepare the spin coherence and to detect its alteration by external perturbations.

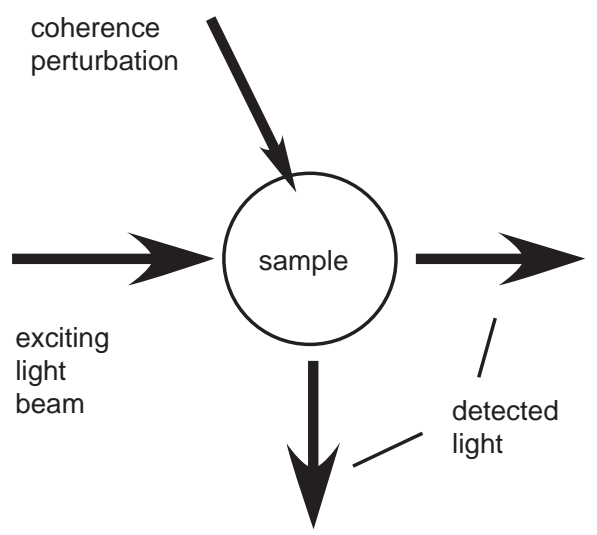

Fig. 1. Principle of optical spin coherence spectroscopy. 
Although in general all the interactions of the sample with the external fields occur simultaneously, the corresponding experiments can often be interpreted in terms of three time-consecutive steps (preparation, evolution, detection) [1]. This interpretation simplifies the mathematical treatment of such processes, in particular for systems with a complex Zeeman substructure. Details of the technique are discussed in a recent review paper of the field of non-linear magneto-optical spectroscopy [2].

The preparation stage involves the process of optical pumping developed in the 1950 s by Kastler and Brossel. Circularly polarized resonance radiation, either from a discharge lamp with suitable spectral filters or from a tunable laser source, transfers angular momentum to the atomic sample (vapor, beam, magneto-optical trap, or doped crystal) via optical absorption-reemission processes. As a consequence of optical pumping the sample becomes spin-polarized. The detection of spin polarization relies on the fact that the optical properties of the medium depend on the magnitude and orientation of its polarization. In many cases the optical absorption coefficient and hence the fluorescence of a $100 \%$ polarized sample vanish. The sample is then called "dark". This feature is used to detect alterations of the spin polarization or coherences by external perturbations. In magnetic resonance, for instance, where an oscillating magnetic field depolarizes a sample previously prepared in a dark state, the fluorescence intensity will show a resonant revival, which can be used to detect the magnetic resonance process. This technique is known as optically-detected magnetic resonance (ODMR). In ODMR one can monitor either fluorescence or transmitted light. The high sensitivity of ODMR can be understood in terms of quantum amplification: the absorption of an audio $(\mathrm{kHz})$ or radio $(\mathrm{MHz})$ frequency quantum by an atom in a dark state allows the absorption (scattering) of one or several optical photons, which corresponds to an energy amplification of the involved quanta by a factor of $10^{8}-10^{10}$ !

As stated above, the resonance linewidth and hence the sensitivity of the process directly depend on the transverse relaxation time $T_{2}$. Unless special precautions are taken, this lifetime in dilute atomic vapors is determined by the time of flight of the atoms from the optical pumping zone to the container walls, where the spin polarization is destroyed by para- or ferro-magnetic impurities in the walls during the atom's adsorption time. Different techniques can be used to lengthen the lifetime. An inert buffer gas may be added to the paramagnetic vapor sample, thereby hindering ballistic trajectories to the walls or/and the walls may be coated by special materials (paraffin, siloxanes) that reduce the sticking time on the wall. Rather than increasing the time of flight to the walls by increasing the sample volume one can increase the size of the containment in one dimension only by realizing an atomic beam. Yet another possibility to prevent depolarizing wall collisions is the freezing of the atoms in a magnetically inert matrix, such as a helium quantum crystal.

\section{Optical magnetometers}

Over the decades many different types and variants of high-sensitivity magnetometric sensors have been developed, for applications ranging from navigation to 
archaeology and geoprospection, from non-destructive testing to medical diagnostics, not to forget their use in fundamental research. Here we address only optically pumped magnetometers (OPM), based on the principle illustrated in Fig. 1, in which the external perturbation may be caused by a static or oscillating magnetic (or electric) field, field gradient, crystal field, collisions, etc. This general definition thus not only covers portable magnetometric devices for field applications, but also larger-scale laboratory equipment, where the optical preparation and detection parts may even be spatially separated as in the Faraday-Ramsey spectrometer discussed below.

Important criteria in the design of a magnetometer apart from its sensitivity are its spatial and its time resolution, the question of whether it is sensitive to the magnitude of the field (scalar type) or just to one of its components (vector type), in which range of magnetic field strengths it can operate, and whether it can be operated in the presence of transverse field components. Other criteria deal with the usability: special operating environments, cooling requirements, mechanical robustness, weight, and power consumption. Here we introduce the basic types of optical magnetometers employed by FRAP, which all make use of optically pumped alkali atoms.

\subsection{The $M_{z}$ magnetometer}

The OPM conceptionally easiest to understand is the so-called $M_{z}$-magnetometer (Fig. 2) [3]. A circularly polarized light beam (from a tunable extended-cavity diode laser in most applications) traverses a sample of alkali atoms (Cs, $\mathrm{Rb}$, or $\mathrm{K})$. The light frequency is tuned to be resonant with an absorption line corresponding to a transition between selected hyperfine levels of the ground $(|g\rangle=|F, M\rangle)$ and excited $(|e\rangle=|f, m\rangle)$ states. For the specific transition shown in Fig. 2 excited atoms returning by emission of fluorescence light to states $|F, M \neq F\rangle$ can be excited again, while those in $|F, M=F\rangle$ remain trapped in that state, due to selection rules for transitions induced by circularly polarized light. As a result of this optical pumping process, all population will end up in the dark state $|F, M\rangle$ after a few cycles. When the sample is exposed to a longitudinal field $B_{0}=B_{z}$ and a transverse field $B_{1}(t)$ oscillating at the frequency $\omega_{\mathrm{rf}}=\omega_{L}$, population will be driven out of the

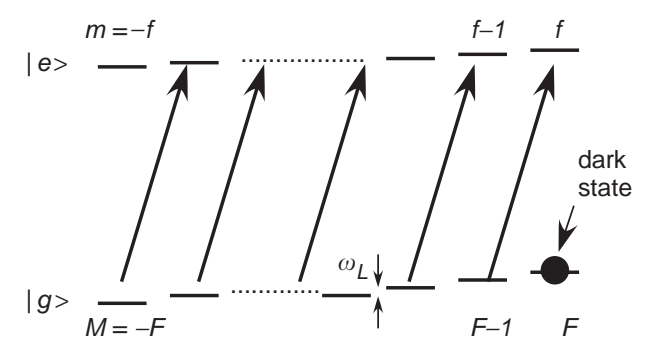

Fig. 2. Preparation of a non-absorbing (dark) state by optical pumping with circularly polarized resonance radiation. 
state $|F, M=F\rangle$, and fluorescence (absorption) will resume. The precise measurement of $\omega_{\text {rf }}$ is thus a measurement of the Larmor frequency $\omega_{L}$ and hence of the field $B_{0}$. The $M_{z}$ magnetometer is a scalar magnetometer, whose resonance frequency is determined by $\left|\overrightarrow{\boldsymbol{B}}_{0}\right|$.

\subsection{The $M_{x}$ magnetometer}

Higher sensitivity can be achieved in the so-called $M_{x}$-configuration, in which the magnetic field subtends an angle ( $45^{\circ}$ for best sensitivity) with the light beam (Fig. 3) [4]. Here again population is optically pumped into the outer Zeeman substate with respect to a quantization direction along the light beam. This optically generated spin polarization $\vec{P}$ precesses around the magnetic-field direction at the Larmor frequency $\omega_{L}$. This precession is resonantly driven by a transverse radio-frequency field $B_{1}(t)$, which leads to a periodic modulation of the optical absorption coefficient at the frequency $\omega_{\mathrm{rf}}$. This modulation can be monitored by detecting either its inphase component, its quadrature component, or its phase with respect to the oscillating radio-frequency field. All three signals show a resonant behavior when $\omega_{\mathrm{rf}}$ is scanned across $\omega_{L}$.

The $M_{x}$-magnetometer is usually operated by actively stabilizing $\omega_{\mathrm{rf}}$ to the Larmor frequency by an electronic feed-back loop, and the magnetometer signal is obtained by measuring the radio frequency $\omega_{\mathrm{rf}}$ in the locked mode. Both the quadrature and the phase signals have a dispersive dependence on detuning and can be used as discriminators providing an error signal for the servo loop. If the phase signal is used, the magnetometer is said to be operated in the phase-locked mode. Alternatively, the oscillating component of the transmission signal can be suitably amplified, phase-shifted, and then fed back to the radio frequency coils, thus making the system oscillate. The advantage of this self-oscillating mode of operation [3]

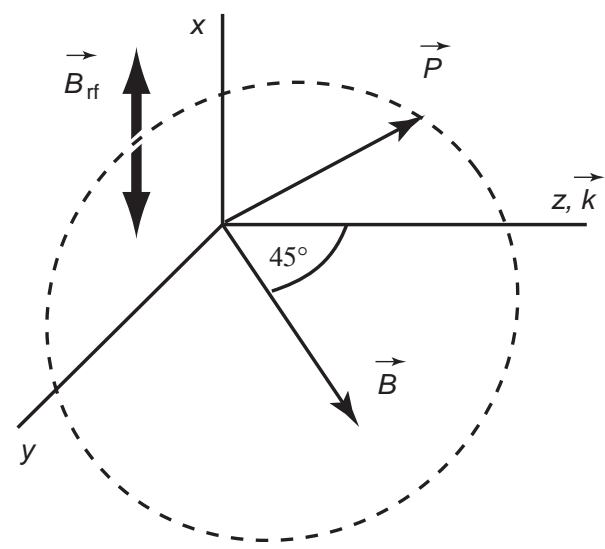

Fig. 3. Configuration of the $M_{x}$-OPM. The spin polarization $\vec{P}$ precesses around the magnetic flux density $\vec{B}$. 
compared to the phase-locked mode is its fast response time, which is ultimately limited by the Larmor frequency only. The $M_{x}$-OPM is also a scalar magnetometer. The superior performance of the $M_{x}$-OPM over the $M_{z}$-OPM stems from its larger signal/noise ratio that can be obtained as only noise contributions in a given bandwidth around the Larmor frequency contribute to the $M_{x}$ signal, while the $M_{z}$ signal is mainly affected by (larger) low-frequency noise components.

\subsection{Faraday-Ramsey spectroscopy}

We have developed another laser-based magnetometric technique, in which a thermal alkali beam interacts with two spatially separated optical pump (preparation) and probe (detection) beams. The resonant circularly or linearly polarized pump beam prepares spin coherences by optical pumping. After evolution in a $30 \mathrm{~cm}$ long interaction zone, in which the atoms are exposed to coherence-changing interactions with magnetic and/or electric fields, the altered coherences are detected by a much weaker probe beam derived from the same laser. The detected signal is either the transmitted power of the probe beam, changes of its polarization, or the fluorescence light that it induces. If a suitably oriented static magnetic field is applied to the interaction zone the resonances observed in this type of magnetometer have an oscillatory Ramsey fringe pattern [5]. Because of the relatively large distribution of thermal velocities only a few pronounced fringes can be observed (Fig. 4). As in some applications the rotation of the probe beam polarization is detected (as in the Faraday effect) we have named this technique Faraday-Ramsey spectroscopy [7]. Because of the small rate of collisions in atomic beams the coherence relaxation time and hence the Ramsey fringe width depends only on the time of flight between the pump and probe zones. For a separation of $30 \mathrm{~cm}$ one observes with cesium atoms a typical fringe width on the order of $100 \mathrm{pT}$. The Faraday-Ramsey technique may be viewed as an extension of the non-linear Faraday effect with spatially separated pump and probe zones [5].

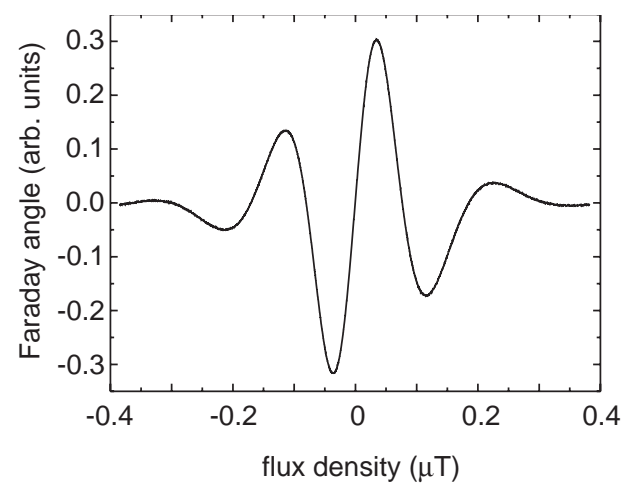

Fig. 4. Typical example of a measured Ramsey signal in a cesium atomic beam with a thermal velocity distribution [6]. 


\section{Applications of optical magnetometry}

\subsection{Magnetocardiography}

Magnetic fields produced by biological organisms contain valuable information on the underlying physiological processes and their pathologies [8]. Currently, superconducting detectors cooled far below room temperature are required to measure these weak biomagnetic signals. Of the many types of optical magnetometers, not all are suited for biomagnetic applications which, besides an ultra-high sensitivity, require simultaneously a time resolution in the millisecond range and a spatial resolution on the cm-scale. This makes the best optical-pumping magnetometer [3] with a sensitivity below $2 \mathrm{fT} / \sqrt{\mathrm{Hz}}$ [4] unsuitable for this application because that sensitivity level can only be obtained with sensor spheres of $15 \mathrm{~cm}$ diameter. Magnetometers based on the non-linear Faraday effect [9] cannot be used either, because they rapidly lose their sensitivity in the presence of transverse field components.

Our biomagnetic research is focused on the detection of human cardiomagnetic fields. We use laser-pumped Cs vapor cells as OPMs, operated in the phase-locked mode described above. In the $M_{x}$-configuration, i.e., with a magnetic bias field at a $45^{\circ}$ angle to the laser propagation direction (Fig. 5a), the intrinsically scalar OPM is mainly sensitive to the cardiomagnetic field component along the bias field, and thus operates as an effective vector magnetometer. The current intrinsic sensitivity of our magnetometer is $80 \mathrm{fT} / \sqrt{\mathrm{Hz}}$ with a cylindrical Cs vapor cell of only $6 \mathrm{~cm}^{3}-\mathrm{a}$ volume 280 times smaller than in [4].

Time-varying stray fields, in particular at the power-line frequency, which are typically many orders of magnitude larger than the strongest peaks of the heart signal, can be efficiently suppressed by a gradiometric arrangement of two (or more)
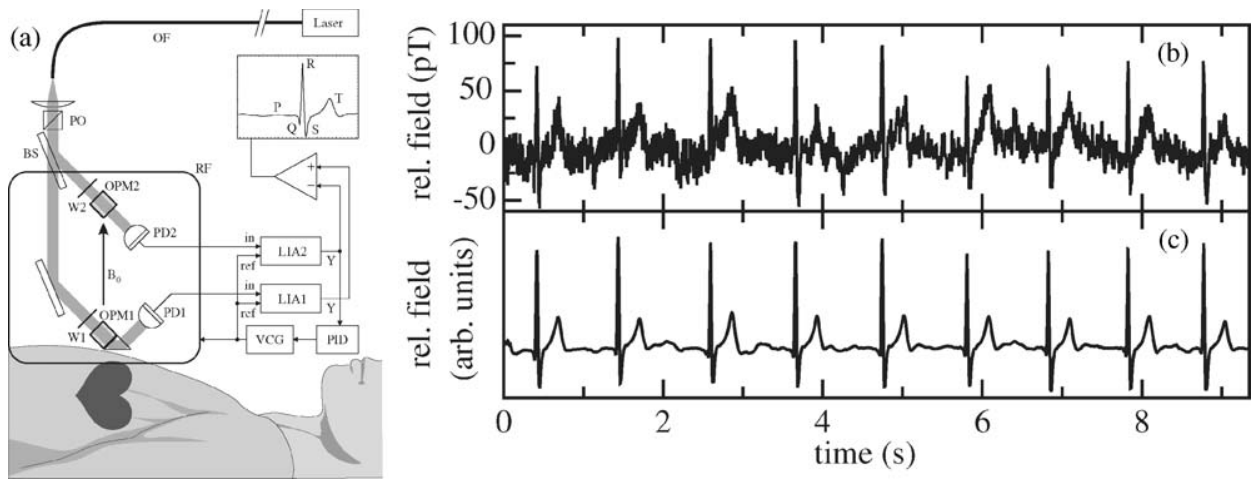

Fig. 5. (a) Experimental setup (OF: optical fiber, PO: polarizer, BS: beam splitter, W: quarter-wave plate, PD: photodiode, RF: coils for the rf field, VCG: voltage-controlled generator, LIA: lock-in amplifier, PID: servo electronics). The inset shows the typical shape and nomenclature of the human magnetocardiogram. (b) Example of the output data of the gradiometer. (c) The same data filtered using state-of-the-art signal recovery techniques [11]. 
sensors [10]. So far we have used one sensor near the chest and a second sensor (OPM2) $7 \mathrm{~cm}$ away as a reference for stray fields, which are then compensated by the servo-loop (Fig. 5a). As a result, sensor OPM1 detects both the heart and stray signals, while OPM2 records stray fields only. In this way a difference measurement allows us to extract the signal of interest, provided that the stray fields have a stable (in)homogeneity. In our first experiments magnetic interference is further suppressed by operating the sensors inside a room weakly shielded by a single layer of mu-metal.

Already the unfiltered output data of the gradiometer (Fig. 5b) allows one to identify the major features of the human magnetocardiogram. After state-of-the-art filtering [11] even the finer features can be resolved (Fig. 5c). Fig. 6 shows a map of the $R$ peak amplitude, measured at 36 points across the chest of one of the authors [12]. Such maps are a useful tool for biomagnetic studies and diagnostics [13]. By averaging traces like those in Fig. $5 \mathrm{~b}$ it is possible to produce a series of such maps covering the whole cardiac cycle, which can then be combined into a movie of the spatial magnetic-field distribution as a function of time [14].

Since a laser OPM should be more than one order of magnitude less expensive to build than a SQUID magnetometer, and since it has basically no operating cost, such devices may have a decisive impact on medical diagnostics in the future. For instance, in a mini-trial with two volunteers, we have compared the maps and movies of the cardiomagnetic field generated both by our optical magnetometer and by a conventional multi-channel SQUID system in the group of Fenici at the Catholic University in Rome [15]. It was found that both methods give basically identical results. In earlier clinical trials cardiological diagnosis based on SQUID-generated

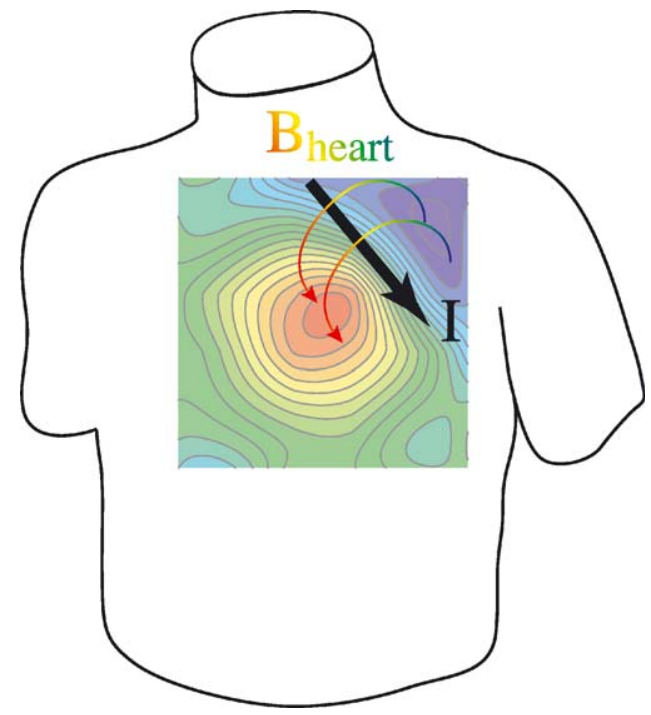

Fig. 6. Map of the $R$ peak of the cardiomagnetic field of one of the authors, with an overlay indicating qualitatively the orientation of the corresponding physiologic current and magnetic field lines at the time of the $R$ peak. 
MCG maps has already proven its worth. We are currently setting up a consortium of physicists, engineers, and cardiologists that will develop and test an improved device, consisting of many parallel second-order gradiometers and associated electronics, packaged for clinical use. We are confident that within 2 to 3 years a practical and affordable device will make the benefits of the harmless and noncontact biomagnetic diagnostic method accessible to everyone, everywhere.

\subsection{Alkali atoms in quantum crystals}

For about one decade our team has devoted a large part of its research efforts to the spectroscopy of atomic defects isolated in crystalline helium matrices. This field of research has been pioneered by our team by the demonstration of implantation techniques [16] and the recording of fluorescence spectra from metal atoms doped into solid helium matrices [17] at temperatures around $1.5 \mathrm{~K}$ and at pressures exceeding 25 bar. While the optical spectra of these samples show strong perturbations (large line shifts and broadenings) due to the helium matrix [18], it was found that the spin polarization [19] and spin coherence [20] of alkali atoms trapped in isotropic body-centered cubic $\mathrm{He}$ crystals were very long-lived with decoherence $\left(T_{2}\right)$ times in the range of several hundred milliseconds [20]. This remarkable property is a consequence of the quantum nature of the helium matrix, which implies that foreign atoms can impose their own symmetry onto the local trapping site by repelling the surrounding helium atoms. Alkali atoms form spherical cavities, called atomic bubbles. Because of their long spin coherence time, atomic bubbles are extremely sensitive to external perturbations. The far-reaching goal of these investigations is the design of a novel technique to search for parity and timereversal violating atomic electric dipole moments [21,22] discussed in the next section.

On the other hand, spin-coherent defect atoms are also very sensitive probes for exploring the symmetries and dynamic interactions with the quantum crystalline surrounding. For instance, when going from the isotropic body-centered cubic phase of the host crystal to its anisotropic hexagonally close-packed configuration, the width of the magnetic resonance spectrum changes by more than three orders of magnitude. This perturbation results from the joint action of electric field gradients and the hyperfine interaction on the spin coherence of the defect atoms. In this way one can measure deformations of the local trapping sites in the hexagonal phase at the level of a few percent [23]. In these investigations, the He matrix-isolated cesium atoms are used both as $M_{z^{-}}$or $M_{x}$-magnetometers with [24] and without [20] phaselocking.

\subsection{Search for permanent electric dipole moments}

It is well known that the discrete symmetries $\mathrm{P}$ (parity conservation) and $\mathrm{T}$ (time reversal invariance) forbid that elementary particles with non-zero spin have a permanent edm. The experimental search for a non-vanishing edm is thus a search for P- and T-violating effects in elementary particles or composite systems, such as 
atoms or molecules [21]. For symmetry reasons a possible edm has to be parallel or antiparallel to the total angular momentum of the system under investigation, so that a change of its orientation by an external electric field will affect the spin coherence and thereby the sample magnetization. Practically all ongoing edm experiments thus look for a change of a magnetic resonance frequency that is proportional to the strength of an externally applied electric field. In this sense edm spectrometers are basically high-sensitivity magnetometers.

FRAP is involved in two edm experiments. On the one hand it is believed that alkali atoms trapped in solid helium (discussed in the previous section) may be promising candidates for performing a sensitive search for atomic edms [22]. The observed long coherence lifetime and corresponding small linewidths of the magnetic resonance signals are prerequisites for a highly sensitive edm search. Another advantage of the matrix-isolated sample is the extremely high electrical break-down voltage in condensed helium, which allows one to apply large electric fields to the samples.

On the other hand, FRAP also participates actively in a collaborative effort aimed at developing a new experiment searching for a permanent edm of the neutron [25]. In that experiment, the precession frequency of ultracold neutrons stored in a container will be measured by a Ramsey resonance technique with two timeconsecutive oscillating field pulses. In the time between these pulses the neutron spins are allowed to precess under the action of parallel or antiparallel magnetic and electric fields. The signature of an edm is a tiny difference of the precession frequencies in these two geometries. A systematic error that could mimic an edm can arise when the magnetic field seen by the neutrons changes by fractions of a picotesla over the course of time intervals of several hundred seconds. In order to control this effect, a special arrangement of $16 \mathrm{Cs}$-OPMs operated in the $M_{x}$-configuration will be used to control and to correct such field fluctuations, down to an effective stability level of only a few femtoteslas. FRAP is responsible for the coordination of the field stabilization activities.

We have developed a laser-pumped Cs-OPM with a $7 \mathrm{~cm}$ diameter paraffin-coated cesium vapor bulb as a sensor for use in the neutron edm experiment. The device is shown in Fig. 7 together with its performance measured inside a three-fold mu-metal shield. The noise level is well above the estimated intrinsic sensitivity of $14 \mathrm{fT} / \sqrt{\mathrm{Hz}}$ and is mainly due to fluctuations of the current source producing the $2-\mu \mathrm{T}$ bias field. In the future we plan to use this feature for the development of an ultra-stable current source starting from a very-well shielded OPM.

\subsection{Faraday-Ramsey spectroscopy}

In the past we have used the Faraday-Ramsey technique introduced above for a quantitative study of the Aharonov-Casher effect [26], a topological phase shift, in a thermal beam of rubidium atoms. The effect manifests itself as a linear Stark effect, which is easily detected as a shift of the Ramsey fringe pattern proportional to a suitably oriented electric field applied to the interaction region. Currently we apply a variant of the Faraday-Ramsey technique for a precision measurement of the 

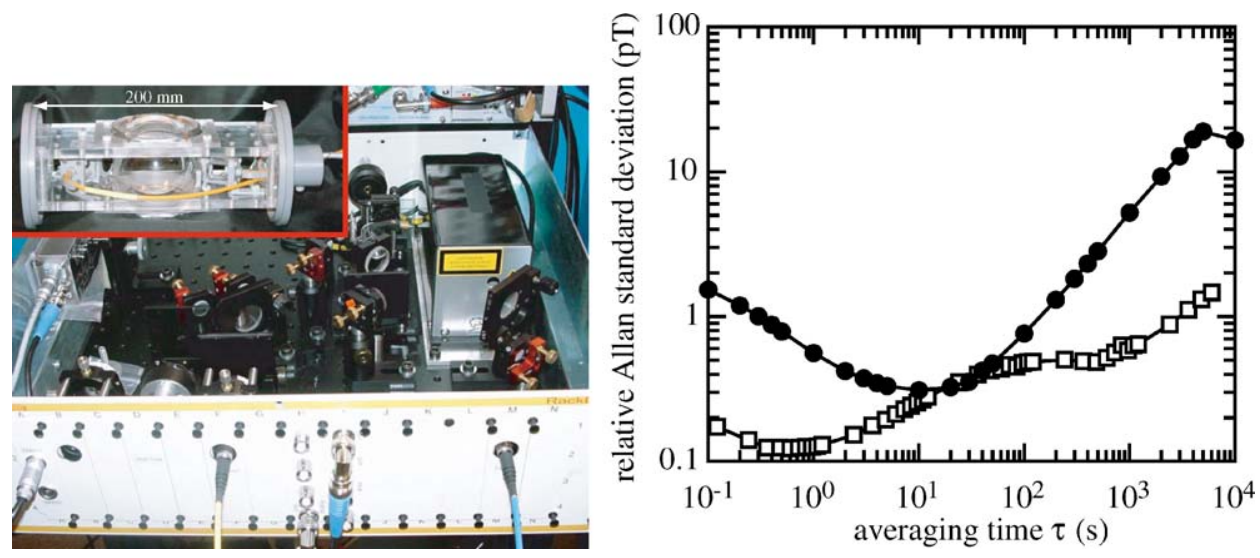

Fig. 7. Left: Setup of one of the sixteen optically pumped magnetometers intended to be used in a neutron edm spectrometer. All the laser optics is contained in a rack-mounted drawer. The inset shows the actual sensor head, made from plastics and glass only. Right: Allan standard deviation of a nominally constant magnetic field measured with the OPM (circles). The squares denote the equivalent field noise due to current instabilities of the power supply for the field coil.

electric tensor polarizabilities $\alpha_{2}$ of alkali atoms [27]. These polarizabilities are forbidden in second-order perturbation theory, but higher-order calculations predict a finite value that is three million times smaller than the well-known scalar polarizability. Optical spin coherence spectroscopy is a most sensitive way to measure this tiny atomic property, which manifests itself via an $M^{2}$-dependent energy shift of Zeeman levels proportional to the square of the applied electric field. Preliminary results for the tensor polarizability of cesium were obtained recently [6]. These results are limited by a systematic effect due to imperfections of the field geometries. A precision redesign of the electrodes and coils will reduce this uncertainty, thereby allowing a measurement of $\alpha_{2}$ with sub-percent accuracy.

\subsection{Spin coherence tomography in the vapor phase}

Two- and three-dimensional magnetic resonance imaging (MRI) is a wellestablished technique in the medical sciences, which was honored by the 2003 Nobel Prize in Medicine. The basic imaging principle relies on the fact that a spin-polarized sample placed in a spatially varying static magnetic field shows a spectrum of magnetic resonance frequencies, which are determined both by the field inhomogeneity and the spatial spin distribution. When the spatial distribution of the field is known (in practice one uses constant gradients) the amplitude distribution of the frequency spectrum allows one to reconstruct the spatial distribution of the spins. By applying field gradients in various spatial directions one can thus obtain threedimensional pictures of the corresponding particle (protons in the case of medical tomography) distributions. Spin polarization is a prerequisite for observing magnetic 
resonance signals. In conventional MRI the spin polarization is determined by the Boltzmann factor in a strong magnetic field.

Inspired by the medical applications and based on our experience with the highsensitivity optical detection of magnetic resonance of vapor atoms we have designed a gas-phase tomograph for the space- and time-resolved study of vapor atoms. Although the spin density in room temperature alkali vapors is more than ten orders of magnitude lower than the proton densities in human tissue, the polarization that can be achieved in such samples by optical pumping is more than five orders of magnitude larger. Moreover, the magnetic fields couple to electronic rather than nuclear magnetic moments, which yields another two to three orders of magnitude in sensitivity. These facts, combined with the highly sensitive detection of the magnetic resonance via the detection of optical photons, has allowed us to observe tomographic signals from vapor phase atoms. As an application we have studied the space- and time-resolved diffusion of cesium atoms in a buffer gas of neon atoms [28]. We have shown that this tomographic technique is a new method for the measurement of diffusion constants, which is free from many of the systematic uncertainties that limit conventional techniques [29].

A pulse of circularly polarized resonant laser radiation in a beam constrained by a $1 \mathrm{~mm}$ diaphragm produces a cylinder of spin-polarized atoms by optical pumping of cesium vapor contained in a cubic quartz cell of $1 \mathrm{~cm}^{3}$ (Fig. 8). The spin-polarized atoms are then allowed to diffuse for a time $\tau$ in a homogeneous magnetic field $B_{z}$ oriented along the polarization. After the delay time $\tau$ a resonant radio-frequency $\pi / 2$-pulse tips the sample magnetization by $90^{\circ}$. The transverse polarization created in this way precesses in the $x-y$ plane while decaying, so that the $P_{x}$-component of the polarization has a damped oscillatory time dependence (free induction decay, FID). The oscillating magnetization is measured via the paramagnetic rotation of the polarization of a second near-resonant probe laser beam. The signal of interest, i.e., the distribution of Larmor frequencies along the probe beam direction, is then
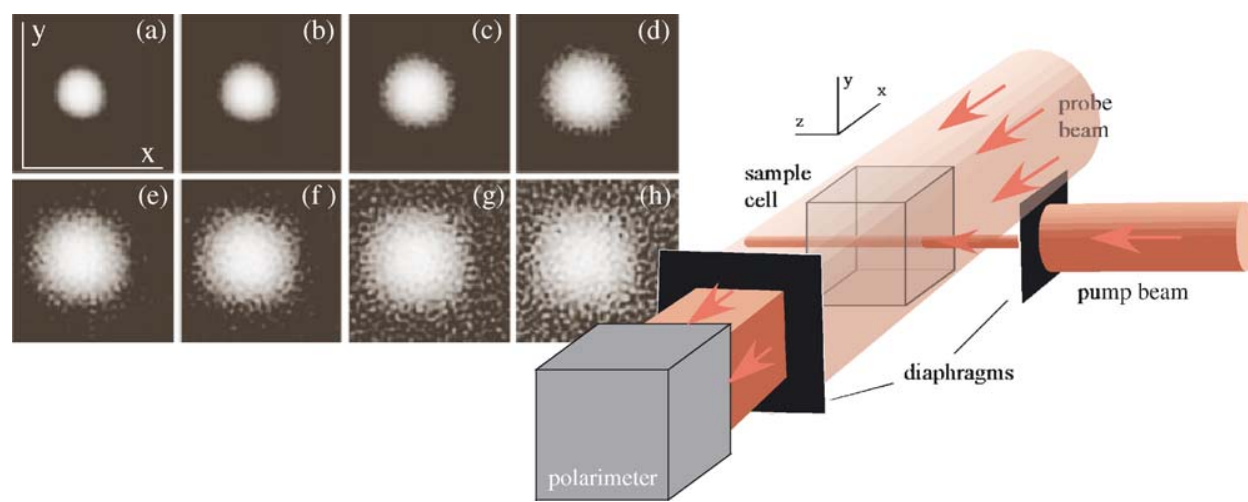

Fig. 8. Arrangement of pump and probe beams for optical magnetic resonance tomography. Coils for homogeneous, oscillating, and gradient fields are not shown. Figs a-h: diffusion of spin-polarized atoms from an initially prepared cylinder, reconstructed from tomographic recordings. Frames are separated by $1 \mathrm{~ms}$. 
inferred by a Fourier transformation of the oscillatory structure. By recording such FID signals for 40 differently oriented field gradients, the two-dimensional spin distribution can be reconstructed. In this way snapshots (Fig. 8) of the distributions during the diffusion process can be obtained by varying the diffusion time $\tau$, which allows a quantitative determination of the diffusion coefficient by a simple analysis.

\section{Conclusion}

The new possibilities offered by laser light sources have opened up new areas of research and application for optical magnetometry. Not only can these magnetometers be used in fundamental research like the hunt for symmetry-violating permanent electric dipole moments in elementary particles, but also do they have a practical advantage for the man in the street, for instance in the form of improved heart diagnostics with the help of laser cardiomagnetometers. In this spirit, we are looking forward to more exciting results in this vigorous field spanning fundamental and applied research.

\section{Acknowledgements}

Some of the results described here have been part of Diploma and $\mathrm{Ph}$. D. Theses by our students. Here we would like to mention and thank only the most recent ones, U. Rasbach, C. Ospelkaus, R. Müller-Siebert, D. Nettels, G. Bison, and S. Gröger. The work described here was supported by grants from the Deutsche Forschungsgemeinschaft (DFG), the Schweizerischer Nationalfonds and from INTAS. R. W. thanks the DFG for financial support through a Heisenberg fellowship.

\section{References}

[1] Weis A, Wurster J, Kanorsky SI. Quantitative interpretation of the non-linear Faraday effect as a Hanle effect of a light-induced birefringence. J Opt Soc Am B 1993;10:716-24.

[2] Budker D, Gawlik W, Kimbal DF, Rochester SM, Yashchuk VV, Weis A. Resonant nonlinear magneto-optical effects in atoms. Rev Mod Phys 2002;74:1153-201.

[3] Bloom AL. Principles of operation of the Rubidium vapor magnetometer. Appl Opt 1962;1:61-8.

[4] Aleksandrov EB, Balabas MV, Vershovskii AK, Ivanov AE, Yakobson NN, Velichanski VL, et al. Laser pumping in the scheme of an $M_{x}$-magnetometer. Opt Spectrosc 1995;78:292-8.

[5] Weis A. Ramseyfication of the resonant nonlinear Faraday effect. In: Figger H, Meschede D, Zimmermann C, editors. Laser physics at the limits. Berlin: Springer; 2001.

[6] Ospelkaus C, Rasbach U, Weis A. An all optical Ramsey resonance technique for the measurement of forbidden tensor polarizabilities. Phys Rev A 2003;67:011402(R)-1-4.

[7] Schuh B, Kanorsky SI, Weis A, Hänsch TW. Observation of Ramsey fringes in nonlinear Faraday rotation. Opt Commun 1993;100:451-5.

[8] Andrä W, Nowak H, editors. Magnetism in medicine. Berlin: Wiley-VCH, 1998.

[9] Budker D, Kimball DF, Rochester SM, Yashchuk VV, Zoloterev M. Sensitive magnetometry based on nonlinear magneto-optical rotation. Phys Rev A 2000;62:043403-1-7. 
[10] Affolderbach C, Stähler M, Knappe S, Wynands R. An all-optical, high-sensitivity magnetic gradiometer. Appl Phys B 2002;75:605-12.

[11] Sternickel K, Effern A, Lehnertz K, Schreiber Th, David P. Nonlinear noise reduction using reference data. Phys Rev E 2001;63:036209-1-4.

[12] Bison G, Wynands R, Weis A. A laser-pumped magnetometer for the mapping of human cardiomagnetic fields. Appl Phys B 2003;76:325-8.

[13] Tavarozzi I, Comani S, Del Gratta C, Di Luzio S, Romani GL, Gallina S, et al. Magnetocardiography: current status and perspectives. Part II: clinical applications. Ital Heart J 2002;3:151-65.

[14] Bison G, Wynands R, Weis A. Dynamical mapping of the human cardiomagnetic field with a roomtemperature laser-optical sensor. Opt Express 2003;11:904-9.

[15] Fenici R, Bison G, Wynands R, Brisinda D, Meloni AM, Weis A. Comparison of magnetocardiographic mapping with SQUID-based and laser-pumped magnetometers in normal subjects. NFSI 2003: 4th International Conference on Noninvasive Functional Source Imaging, Chieti, Italy, September 10-13, 2003.

[16] Arndt M, Dziewior R, Kanorsky S, Weis A, Hänsch TW. Implantation of metal atoms into solid helium. Z Phys B 1995;98:377-81.

[17] Kanorsky SI, Arndt M, Dziewior R, Weis A, Hänsch TW. Optical Spectroscopy of atoms trapped in solid helium. Phys Rev B 1994;49:3645-7.

[18] Kanorsky SI, Arndt M, Dziewior R, Weis A, Hänsch TW. Pressure shift and broadening of the resonance line of Barium atoms trapped in liquid helium. Phys Rev B 1994;50:6296-302.

[19] Arndt M, Kanorsky SI, Weis A, Hänsch TW. Long electronic spin relaxation times of Cs atoms in solid ${ }^{4}$ He. Phys Rev Lett 1995;74:1359-62.

[20] Kanorsky SI, Lang S, Lücke S, Ross SB, Hänsch TW, Weis A. Millihertz magnetic resonance spectroscopy of Cs atoms in body-centered-cubic ${ }^{4} \mathrm{He}$. Phys Rev A 1996;54:R1010-3.

[21] Khriplovich IB, Lamoreaux SK. CP violation without strangeness. Berlin: Springer; 1997.

[22] Weis A, Kanorsky S, Arndt M, Hänsch TW. Spin physics in solid helium: experimental results and applications. Z Phys B 1995;98:359-62.

[23] Kanorsky SI, Lang S, Eichler T, Winkler K, Weis A. Quadrupolar deformations of atomic bubbles in solid ${ }^{4}$ He. Phys Rev Lett 1998;81:401-4.

[24] Nettels D, Müller-Siebert R, Huang X, Ulzega S, Weis A. Magnetic and electric properties of alkali atoms trapped in solid helium. Physica B 2003;329:406-8.

[25] Aleksandrov E, Balabas M, Ban G, Bison G, Bodek K, Borisov Yu, et al. Search for a neutron electric dipole moment: progress report. PSI Scientific Report 2002, vol. I, 2003. p. 25.

[26] Görlitz A, Schuh B, Weis A. Measurement of the Aharonov-Casher phase of aligned Rb atoms. Phys Rev A 1995;51:R4305-8.

[27] Angel JRP, Sandars PGH. The hyperfine structure Stark effect. I. Theory. Proc Roy Soc A 1968:305:125-38.

[28] Giel D, Nettels D, Weis A. Imaging of spin diffusion in the vapour phase by optical magnetic resonance tomography. ICOLS99: XIV International Conference on Laser Spectroscopy. Innsbruck, Austria, June 7-10, 1999.

[29] Giel D, Hinz G, Nettels D, Weis AR. Diffusion of Cs atoms in Ne buffer gas measured by optical magnetic resonance tomography. Opt Express 2000;6:251-6. 